\title{
Charged Massive Particle's Tunneling from Charged Nonrotating Microblack Hole
}

\author{
M. J. Soleimani, ${ }^{1}$ N. Abbasvandi, ${ }^{2}$ Shahidan Radiman, ${ }^{2}$ and W. A. T. Wan Abdullah ${ }^{1}$ \\ ${ }^{1}$ Department of Physics, University of Malaya, 50603 Kuala Lumpur, Malaysia \\ ${ }^{2}$ School of Applied Physics, FST, University Kebangsaan Malaysia, 43600 Bangi, Malaysia
}

Correspondence should be addressed to M. J. Soleimani; msoleima@cern.ch

Received 30 March 2016; Revised 23 June 2016; Accepted 26 June 2016

Academic Editor: Barun Majumder

Copyright (c) 2016 M. J. Soleimani et al. This is an open access article distributed under the Creative Commons Attribution License, which permits unrestricted use, distribution, and reproduction in any medium, provided the original work is properly cited. The publication of this article was funded by SCOAP ${ }^{3}$.

In the tunneling framework of Hawking radiation, charged massive particle's tunneling in charged nonrotating TeV-scale black hole is investigated. To this end, we consider natural cutoffs as a minimal length, a minimal momentum, and a maximal momentum through a generalized uncertainty principle. We focus on the role played by these natural cutoffs on the luminosity of charged nonrotating microblack hole by taking into account the full implications of energy and charge conservation as well as the backscattered radiation.

\section{Introduction}

One of the most exciting consequences of models of low scale gravity [1-4] is the possibility of production of small black holes [5-7] at particle colliders such as the Large Hadron Collider (LHC) as well as in Ultrahigh Energy Cosmic Ray Air Showers (UECRAS) [8-10]. Incorporation of gravity in quantum field theory supports the idea that the standard Heisenberg uncertainty principle should be reformulated by the so-called generalized uncertainty principle near the Planck scale [11-13]. In particular, the existence of a minimum observable length is indicated by string theory [14], TeVscale black hole physics [15], and loop quantum gravity [16]. Moreover, some black hole Gedanken experiments support the idea of existence of a minimal measurable length in a fascinating manner $[17,18]$ On the other hand, Doubly Special Relativity theories [19-24] suggest that a test particle's momentum cannot be arbitrarily imprecise and there is an upper bound for momentum fluctuation. It means that there is also a maximal particle momentum. It has been shown that incorporation of quantum gravity effects in black hole physics and thermodynamics through a generalized uncertainty principle (GUP) with the mentioned natural cutoffs modifies the result dramatically, specially, the final stage of black hole evaporation. Parikh and Wilczek on their pioneering work [25] constructed a procedure to describe the Hawking radiation emitted from a Schwarzschild black hole as a tunneling through its quantum horizon. The emission rate (tunneling probability) which arising from the reduction of the black hole mass is related to the change of black hole entropies before and after the emission. In this paper, charged particle's tunneling from charged nonrotating microblack hole is investigated. We consider a more general framework of GUP that admits a minimal length, minimal momentum, and maximal momentum to study the effects of natural cutoffs on the tunneling mechanism and luminosity of charged nonrotating $\mathrm{TeV}$-scale black holes with extra dimensions in Arkani-Hamed, Dimopoulos, and Dvali (ADD) model [1] in the context of this GUP. The calculation shows that the emission rate satisfies the first law of black hole thermodynamics. The paper is organized as follows: in Section 2, we introduce a generalized uncertainty principle with minimal length, minimal momentum, and maximal momentum. In Section 3, we obtain an expression for emission rate of charged particle from charged nonrotating microblack hole based on the ADD model and the mentioned GUP. We consider the backscattering of the emitted radiation taking into account energy and charge conservation to evaluate the luminosity of $\mathrm{TeV}$-scale black hole in presence of natural cutoffs. The last part is the discussion and calculation. 


\section{Generalized Uncertainty Principle}

The existence of a minimal measurable length of the order of the Planck length, $l_{p} \sim 10^{-35} \mathrm{~m}$, was indicated by most of quantum gravity approaches $[26,27]$ which modifies the Heisenberg uncertainty principle (HUP) to the so-called generalized (gravitational) uncertainty principle (GUP). The minimal position uncertainty, $\Delta x_{0}$, could be not made arbitrarily small toward zero [12] in the GUP framework due to its essential restriction on the measurement precision of the particle's position. On the other hand, Doubly Special Relativity (DSR) theories [21-24] have considered that existence of a minimal measurable length would restrict a test particle's momentum to take arbitrary values and therefore there is an upper bound for momentum fluctuation [28, 29]. So there is a maximal particle's momentum due to the fundamental structure of space-time at the Planck scale [30, 31]. Based on the above arguments, the GUP that predicts both a minimal length and a maximal momentum can be written as follows $[19,20]$ :

$$
\Delta x \Delta p \geq \frac{\hbar}{2}\left[1-\alpha\langle\Delta p\rangle+2 \alpha^{2}\langle\Delta p\rangle^{2}\right]
$$

The relation (1) can lead us to the following commutator relation:

$$
[x, p]=i \hbar\left(1-\alpha p+2 \alpha^{2} p^{2}\right)
$$

where $\alpha$ is GUP dimensionless positive constant of both minimal length and maximal momentum that depends on the details of the quantum gravity hypothesis. It has been developed that particle's momentum cannot be zero if the curvature of space-time becomes important and its effects are taken into account $[32,33]$. In fact, there appears to be a limit to the precision of which the corresponding momentum can be expressed as a nonzero minimal uncertainty in momentum measurement. Based on this more general framework as a consequence of small correction to the canonical commutation relation, this GUP can be represented as [34]

$$
\Delta x \Delta p \geq \hbar\left(1-\alpha l_{p} \Delta p+\alpha^{2} l_{p}^{2}(\Delta p)^{2}+\beta^{2} l_{p}^{2}(\Delta x)^{2}\right)
$$

which in extra dimensions can be written as follows:

$$
\begin{aligned}
& \Delta x_{i} \Delta p_{i} \\
& \quad \geq \hbar\left(1-\alpha l_{p}\left(\Delta p_{i}\right)+\alpha^{2} l_{p}^{2}\left(\Delta p_{i}\right)^{2}+\beta^{2} l_{p}^{2}\left(\Delta x_{i}\right)^{2}\right) .
\end{aligned}
$$

Here, $\alpha$ and $\beta$ are dimensionless positive coefficients which are independent of $\Delta x$ and $\Delta p$. In general they may depend on expectation value of $x$ and $p$. According to the generalized Heisenberg algebra, we suppose that operators of position and momentum obey the following commutation relation:

$$
[x, p]=i \hbar\left(1-\alpha p+\alpha^{2} p^{2}+\beta^{2} x^{2}\right) \text {. }
$$

In what follows, we use this more general framework of GUP to find the tunneling rate of emitted particles through charged nonrotating $\mathrm{TeV}$-scale black holes.

\section{Tunneling Mechanism}

The idea of large extra dimensions might allow studying interactions at Trans-Planckian energies in particle colliders and the ADD model used $d$ new large space-like dimensions. So, in order to investigate the Hawking radiation via tunneling from charged nonrotating $\mathrm{TeV}$-scale black holes of higher dimensional, a natural candidate is that of ReissnerNordstrom $d$-dimensional modified solution in presence of generalized uncertainty principle. In this case, the line element of $d$-dimensional Reissner-Nordstrom solution of Einstein field equation is given by [35]

$$
\begin{aligned}
d s^{2} & =f(r) c^{2} d t^{2}-f^{-1}(r) d r^{2}-r^{2} d \Omega_{d-2}^{2} \\
& =g_{\mu \nu} d x^{\mu} d x^{\nu}
\end{aligned}
$$

where $\Omega_{d-2}$ is the metric of the unit $S^{d-2}$ as $\Omega_{d-2}=$ $2 \pi^{(d-1) / 2} / \Gamma((d-1) / 2)$ and

$$
f=f(M, Q, r)=1-\frac{\omega_{d-2} M}{r^{d-3}}+\frac{\omega_{d-2} Q^{2}}{2(d-3) \Omega_{d-2} r^{2(d-3)}},
$$

where $\omega_{d-2}=16 \pi /(d-2) \Omega_{d-2}$. Here $M$ and $Q$ are the mass and electric charge of the black hole, respectively; units $G_{d}=$ $c=\hbar=1$ are adopted throughout this paper. The black hole has an outer/inner horizon located at

$$
r_{ \pm}^{d-3}=\frac{\omega_{d-2}}{2}\left[M \pm \sqrt{M^{2}-\frac{(d-2) Q^{2}}{8 \pi(d-3)}}\right] .
$$

Therefore, the event horizon shrinks, and the inner one appears; when the black hole becomes charged the inner radius is related to the amount of charge and the outer one $r_{+}$corresponds to the radius of Schwarzschild black hole. In this case, (8) can be rewritten as follows:

$$
r_{+}=\left(\frac{\omega_{d-2}}{2}\left[M+\sqrt{M^{2}-\frac{(d-2) Q^{2}}{8 \pi(d-3)}}\right]\right)^{1 /(d-3)} .
$$

In order to apply the semiclassical tunneling analysis, one can find a proper coordinate system for the black hole metric where all the constant lines are flat and the tunneling path is free of singularities. In this manner, Painlevé coordinates are suitable choices. In these coordinates, the $d$-dimensional Reissner-Nordstrom metric is given by

$$
d s^{2}=-f d t^{2} \pm 2 \sqrt{1-f} d t d r+d r^{2}+r^{2} d \Omega_{d-2}^{2}
$$

which is stationary, nonstatic, and nonsingular at the horizon and plus (minus) sign corresponds to the space-time line element of the outgoing (incoming) particles across the event horizon, respectively. The trajectory of charged massive particles as a sort of de Broglie s-wave can be approximately determined as $[36,37]$

$$
\dot{r}=\frac{d r}{d t}=-\frac{g_{t t}}{2 g_{t r}}= \pm \frac{f}{2 \sqrt{1-f}},
$$


where the plus (minus) sign denotes the radial geodesics of the outgoing (incoming) charged particles tunneling across the event horizon, respectively. We incorporate quantum gravity effects in the presence of the minimal length, minimal momentum, and maximal momentum via the GUP which motivates modification of the standard dispersion relation in the presence of extra dimensions based on ADD model. If the GUP is a fundamental outcome of quantum gravity proposal, it should appear that the de Broglie relation is as follows:

$$
\begin{aligned}
& \lambda_{ \pm} \\
& =\frac{p_{i}}{2 \beta^{2} l_{p}^{2}}\left(1 \pm \sqrt{1-\frac{4 \beta^{2} l_{p}^{2}\left(1-\alpha l_{p} p_{i}+\alpha^{2} l_{p}^{2} p_{i}^{2}\right)}{p_{i}^{2}}}\right) .
\end{aligned}
$$

One can find easily that positive sign does not recover ordinary relation in the limits $\alpha \rightarrow 0$ and $\beta \rightarrow 0$. So we consider the minus sign as

$$
\begin{aligned}
\lambda_{-}= & \left(\frac{3 \beta^{2} l_{p}^{4}}{p_{i}}+p_{i} l_{p}^{2}\right) \alpha^{2}-\left(\frac{2 \beta^{2} l_{p}^{3}}{p_{i}^{2}}+l_{p}\right) \alpha+\frac{\beta^{2} l_{p}^{2}}{p_{i}^{3}} \\
& +\frac{1}{p_{i}}
\end{aligned}
$$

or equivalently

$$
\begin{aligned}
\varepsilon= & \left(3 E \beta^{2} l_{p}^{4}+E^{3} l_{p}^{2}\right) \alpha^{2}-\left(2 \beta^{2} l_{p}^{3}+E^{2} l_{p}\right) \alpha+E \\
& +\frac{\beta^{2} l_{p}^{2}}{E} .
\end{aligned}
$$

Here, for investigating Hawking radiation of charged massive particles from the event horizon of charged nonrotating microblack hole, we use this more general uncertainty principle and take into consideration the response of background geometry to radiated quantum of energy $E$ with GUP correction, that is, $\varepsilon$. The emitted particle which can be treated as a shell of energy $\varepsilon$ and charge $q$ moves on the geodesics of a space-time with central mass $M-\varepsilon$ substituted for $M$ and charge parameter $Q-q$ replaced with $Q$. We set the total Arnowitt-Deser-Misner (ADM) mass, $M$, and the ADM charge of the space-time to be fixed but allow the hole mass and charge to fluctuate and replace $M$ by $M-\varepsilon$ and $Q$ by $Q-q$ in both the metric and the geodesic equation. So the outgoing radial geodesics of the charged massive particle tunneling out from the event horizon and the nonzero component of electromagnetic potential are

$$
\begin{aligned}
\dot{r} & =\frac{f(M-\varepsilon, Q-q, r)}{2 \sqrt{1-f(M-\varepsilon, Q-q, r)}}, \\
A_{t} & =\frac{Q-q}{(d-3) \Omega_{d-2} r^{d-3}} .
\end{aligned}
$$

So the Lagrangian for the matter-gravity system is

$$
L=L_{m}+L_{e}
$$

where $L_{e}=-(1 / 4) F_{\mu \nu} F^{\mu \nu}$ is the Lagrangian function of the electromagnetic field corresponding to the generalized coordinates $A_{\mu}=\left(A_{t}, 0,0,0\right)$ [38].

We assume the tunneling mechanism as a semiclassical method producing Hawking radiation. In this case, using $\mathrm{WKB}$ approximation, the emission rate of tunneling massive charged particle can be obtained from the imaginary part of the particle action at the stationary phase for the tunneling trajectory; namely, [39, 40]

$$
\Gamma \sim \exp (-2 \operatorname{Im} I)
$$

Assuming the generalized coordinate $A_{t}$ is an ignorable one, to eliminate this degree of freedom completely, we can obtain the action of the matter-gravity system as

$$
\begin{aligned}
I & =\int_{t_{i}}^{t_{f}}\left(L-P_{A_{t}} \dot{A}_{t}\right) d t \\
& =\int_{r_{i}}^{r_{f}}\left[\int_{(0,0)}^{\left(p_{r}, p_{A_{t}}\right)}\left(\dot{r} d p^{\prime}{ }_{r}-\dot{A}_{t} d p_{A_{t}}^{\prime}\right)\right] \frac{d r}{\dot{r}},
\end{aligned}
$$

where $r_{i}$ and $r_{f}$ are the location of the event horizon corresponding to $t_{i}$ and $t_{f}$, respectively, before and after the particle of energy $\varepsilon$ and charge $q$ tunnels out, in which $p_{A_{t}}$ and $p_{r}$ are the canonical momentum conjugate to the coordinates $A_{t}$ and $r$, respectively.

In order to consider the effect of quantum gravity, the commutation relation between the radial coordinate components and conjugate momentums should be modified based on (1) and (2) of the expressed GUP as follows [41]:

$$
\left[r, p_{r}\right]=i\left(1-\alpha l_{p} p_{r}+\alpha^{2} l_{p}^{2} p_{r}^{2}\right)
$$

So as it is clear from the more general GUP and based on (5) the commutation relation should be modified as

$$
\left[r, p_{r}\right]=i\left(1-\alpha l_{p} p_{r}+\alpha^{2} l_{p}^{2} p_{r}^{2}+\beta^{2} l_{p}^{2} r^{2}\right)
$$

In the classical limit it is replaced by Poisson bracket as follows:

$$
\left\{r, p_{r}\right\}=\left(1-\alpha l_{p} p_{r}+\alpha^{2} l_{p}^{2} p_{r}^{2}+\beta^{2} l_{p}^{2} r^{2}\right)
$$

Now, we apply the deformed Hamiltonian equation:

$$
\begin{aligned}
& \dot{r}=\{r, H\}=\left.\left\{r, p_{r}\right\} \frac{d H}{d r}\right|_{r}, \\
& \qquad\left.d H\right|_{\left(r, A_{t}, p_{t}\right)}=d(M-\varepsilon) \\
& \dot{A}_{t}=\frac{d H}{d p_{A_{t}}}=\frac{d E_{Q}^{\prime}}{d p_{A_{t}}},\left.\quad d H\right|_{\left(A_{t}, r, p_{r}\right)}=A_{t} d(Q-q) .
\end{aligned}
$$

In (18) as the Hamiltonian is $H=M-\varepsilon^{\prime}$, one can set $p^{2} \simeq$ $\varepsilon^{\prime 2}$ and $p \simeq \varepsilon^{\prime}$ and eliminate the momentum in favor of the energy in integral (18) and switching the order of integration yields the imaginary part of the action as follows: 


$$
\begin{aligned}
& \operatorname{Im} I=\operatorname{Im} \int_{r_{i}}^{r_{f}} \int_{(M, Q)}^{(M-\varepsilon, Q-q)}\left[\left(1-\alpha l_{p} \varepsilon^{\prime}+\alpha^{2} l_{p}^{2} \varepsilon^{\prime 2}+\beta l_{p}^{2} r^{2}\right) d\left(M-\varepsilon^{\prime}\right)-\frac{Q-q^{\prime}}{(d-3) \Omega_{d-2} r^{d-3}} d\left(Q-q^{\prime}\right)\right] \frac{d r}{\dot{r}} \\
& =\operatorname{Im} \int_{r_{i}}^{r_{f}} \int_{(M, Q)}^{(M-\varepsilon, Q-q)} \frac{2 \sqrt{1-f\left(M-\varepsilon^{\prime}, Q-q^{\prime}, r\right)}}{f\left(M-\varepsilon^{\prime}, Q-q^{\prime}, r\right)}\left[\left(1-\alpha l_{p} \varepsilon^{\prime}+\alpha^{2} l_{p}^{2} \varepsilon^{\prime 2}+\beta l_{p}^{2} r^{2}\right) d\left(M-\varepsilon^{\prime}\right)-\frac{Q-q^{\prime}}{(d-3) \Omega_{d-2} r^{d-3}} d\left(Q-q^{\prime}\right)\right] d r .
\end{aligned}
$$

$r$ integral can be evaluated by deforming the contour of the single pole at the outer horizon. During $r$ integral first, we find

$$
\begin{gathered}
\operatorname{Im} I=\operatorname{Im} \int_{0}^{\varepsilon} 2(-\pi i) r_{+}\left(M-\varepsilon^{\prime}, Q-q\right) \\
\cdot\left(1-\alpha l_{p} \varepsilon^{\prime}+\alpha^{2} l_{p}^{2} \varepsilon^{\prime 2}+\beta l_{p}^{2}\right) d\left(-\varepsilon^{\prime}\right) \\
-\int_{0}^{Q-q} 2(-\pi i) r_{+}\left(M-\varepsilon, Q-q^{\prime}\right) \\
\cdot \frac{\left(Q-q^{\prime}\right)}{(d-3) \pi r^{d-3}} d\left(Q-q^{\prime}\right) .
\end{gathered}
$$

This allows us to consider the leading order correction to be just proportional to second order of $\alpha l_{p}$ and also second order of $\beta l_{p}$ for simplicity without loss of generality. In this regard, we can finish the integration by applying Taylor series and obtain the imaginary part of the action. Although integral
(24) is complicated, one can find such terms as an example for $d=5$ as follows:

$$
\begin{aligned}
& \operatorname{Im} I_{d=5} \approx \cdots+4096 \alpha l_{p} \pi M^{2}-8192 \alpha l_{p} \pi M\left(3 E \beta^{2} l_{p}^{4}\right. \\
& \left.+E^{3} l_{p}^{2}\right) \alpha^{2}+8192 \alpha l_{p} \pi M\left(2 \beta^{2} l_{p}^{3}+E^{2} l_{p}\right) \alpha \\
& \quad-8192 \alpha l_{p} \pi M E-8192 \alpha l_{p} \pi M \frac{\beta^{2} l_{p}^{2}}{E} \\
& +4096 \alpha l_{p} \pi\left(\left(3 E \beta^{2} l_{p}^{4}+E^{3} l_{p}^{2}\right) \alpha^{2}\right. \\
& \left.\quad-\left(2 \beta^{2} l_{p}^{3}+E^{2} l_{p}\right) \alpha+E+\frac{\beta^{2} l_{p}^{2}}{E}\right)^{2}-192 \alpha l_{p} Q^{2} \\
& +\cdots
\end{aligned}
$$

Substituting (24) into (17), the tunneling probability of charged particles from charged nonrotating $\mathrm{TeV}$-scale black holes is obtained as

$$
\begin{aligned}
\Gamma & =\exp (-2 \operatorname{Im} I) \simeq \exp \left[\operatorname{Im} \int_{0}^{\varepsilon} 2(-\pi i) r_{+}\left(M-\varepsilon^{\prime}, Q-q\right)\left(1-\alpha l_{p} \varepsilon^{\prime}+\alpha^{2} l_{p}^{2} \varepsilon^{\prime 2}+\beta l_{p}^{2}\right) d\left(-\varepsilon^{\prime}\right)\right. \\
& \left.-\int_{0}^{Q-q} 2(-\pi i) r_{+}\left(M-\varepsilon, Q-q^{\prime}\right) \frac{\left(Q-q^{\prime}\right)}{(d-3) \pi r^{d-3}} d\left(Q-q^{\prime}\right)\right]=\exp (\Delta s),
\end{aligned}
$$

where $\Delta s$ is the difference in black hole entropies before and after emission [42-47]. It was shown that the emission rates on the high energy scales correspond to differences between the counting of states in the microcanonical and in the canonical ensembles $[48,49]$. By performing integration on (24), one can find that the first order of $E$ in the exponential gives a thermal, Boltzmannian spectrum. The existence of extra terms in relation (25) shows that the radiation is not completely thermal. In fact, these extra terms enhance the nonthermal character of the radiation. Also, it is easy to find that the tunneling rate should be greater than the ordinary one in any stage of tunneling process. This tunneling rate compared to the tunneling rate which is calculated in [50] obviously shows that, by considering all natural cutoffs in generalized uncertainty principle relation, many additional terms appeared. The additional terms show strong deviation of microblack holes radiation from ordinary thermal radiation.

\section{Backscattering and Luminosity}

It has been shown [51] that black holes radiate a thermal spectrum of particles. So microblack holes emit black body radiation at the Hawking temperature. Following a heuristic argument [52], the energy of the Hawking particles is $\Delta E \approx$ $c \Delta p$ and it is deduced for the Hawking temperature of black hole based on LED scenario,

$$
T_{H} \simeq \frac{(d-3) \Delta p}{4 \pi},
$$

where $(d-3) / 4 \pi$ is a calibration factor in $d$-dimensional space-time. By saturating inequality (4), one can find 
momentum uncertainty in terms of position uncertainty as follows:

$$
\begin{aligned}
\Delta P_{i}= & \left(\frac{\alpha l_{p}+\Delta x_{i}}{4 \alpha^{2} l_{p}^{2}}\right) \\
& \cdot\left(1 \pm \sqrt{1-\frac{4 \alpha^{2} l_{p}^{2}\left(1+4 \beta^{2} l_{p}^{2}\left(\Delta x_{i}\right)^{2}\right)}{\left(\alpha l_{p}+\Delta x_{i}\right)^{2}}}\right) .
\end{aligned}
$$

So the modified black hole Hawking temperature in the presence of natural cutoffs becomes

$$
\begin{aligned}
T_{H} & =\frac{(d-3)\left(2 r_{+}+\alpha l_{p}\right)}{16 \pi \alpha^{2} l_{p}^{2}}(1 \\
& -\sqrt{\left.1-\frac{4 \alpha^{2} l_{p}^{2}\left(1+16 \beta^{2} l_{p}^{2} r_{+}{ }^{2}\right)}{\left(2 r_{+}+\alpha l_{p}\right)^{2}}\right)} .
\end{aligned}
$$

Based on (29), GUP give rise to the existence of a minimal mass of a charged nonrotating microblack hole given by

$$
\begin{aligned}
& M_{\mathrm{min}}^{\mathrm{GUP}} \\
& =\frac{(d-2) \Omega}{16 \pi}\left(\left(\frac{\left(1+2 \sqrt{1-12 \beta^{2} \alpha^{2} L_{p l}^{4}}\right) L_{p l} \alpha}{32 \beta^{2} \alpha^{2} L_{p l}^{4}-2}\right)^{2 d-6}\right. \\
& \left.+\frac{8 \pi Q^{2}}{(d-2)(d-3)}\right) \\
& \cdot\left(\left(\frac{\left(1+2 \sqrt{1-12 \beta^{2} \alpha^{2} L_{p l}^{4}}\right) L_{p l} \alpha}{32 \beta^{2} \alpha^{2} L_{p l}^{4}-2}\right)^{d-3}\right)^{-1} .
\end{aligned}
$$

Therefore, there are some black hole remnants without radiation based on (30). A radiated particle state corresponding to an arbitrary finite number of virtual pairs inside the black hole event horizon is as follows [53]:

$$
|\psi\rangle=N \sum e^{-\pi n \varepsilon / \hbar k}\left|n_{\text {out }}^{(L)}\right\rangle \otimes\left|n_{\text {out }}^{(R)}\right\rangle
$$

where $N^{2}=e^{\gamma \varepsilon} /\left(e^{\gamma \varepsilon}-1\right)$ is a normalization constant and $k$ is the surface gravity. This quantum state is transformed with respect to an observer outside the horizon. In order to obtain the average particle number in the energy state $\varepsilon$ with respect to an observer, one can trace out the inside degrees of freedom to yield the reduced density matrix of the form

$$
\begin{aligned}
\rho_{\text {reduced }}= & \left(1-\exp \left(-\frac{2 \pi \varepsilon}{\hbar k}\right)\right) \sum_{n=0}^{\infty} e^{-\gamma n \varepsilon}\left|n_{\text {out }}^{(R)}\right\rangle \\
& \otimes\left\langle n_{\text {out }}^{(R)}\right| .
\end{aligned}
$$

In this regard, the number distribution with respect to $\varepsilon$ is given by

$$
\left\langle n_{\varepsilon}\right\rangle=\operatorname{trace}\left(n \rho_{\text {reduced }}\right)=\frac{1}{e^{\gamma \varepsilon}-1},
$$

where $\gamma=1 / T_{H}$. Whenever a particle is radiated from the microblack hole event horizon, its wave function satisfies a wave equation with an effective potential that depends on outer event horizon. As the potential represents a barrier to the outgoing radiation, one part of the radiation is backscattered.

In this way, it can be shown that the distribution $\left\langle n_{\varepsilon}\right\rangle$ for the Hawking radiation will be modulated by grey body factor [54] which for a charged nonrotating TeV-scale black hole is given as

$$
\Lambda=4 \varepsilon^{2} r_{+}^{2}
$$

which $\Lambda$ is the standard approximated grey body factor. In this way, one can take energy and charge conservation into account [54] and get the straightforward result by substituting (14) into (34). So we obtain

$$
\begin{aligned}
& \Lambda_{E C}=4\left[E\left(1-\alpha l_{p} E+\alpha^{2} l_{p}^{2} E^{2}\right)\right. \\
& \left.\quad\left(1+\frac{\beta^{2} l_{p}^{2}}{E^{2}}\left(1-\alpha l_{p} E+\alpha^{2} l_{p}^{2} E^{2}\right)\right)\right]^{2} r_{+}^{2}(M-\varepsilon, Q \\
& -q) .
\end{aligned}
$$

On the other hand, if we consider the full consequences of energy and charge conservation, for total flux, including backscattering [55], the luminosity modulated according to the grey body factor has to be written as

$$
\begin{aligned}
L^{d}(M) & =\frac{1}{2 \pi} \int_{0}^{M-M_{\min }^{\mathrm{GUP}}}\left\langle n_{\varepsilon}\right\rangle \Lambda_{E C} \varepsilon d \varepsilon \\
& =\frac{1}{2 \pi} \int_{0}^{M-M_{\min }^{\mathrm{GUP}}} \frac{4\left[E\left(1-\alpha l_{p} E+\alpha^{2} l_{p}^{2} E^{2}\right)\left(1+\left(\beta^{2} l_{p}^{2} / E^{2}\right)\left(1-\alpha l_{p} E+\alpha^{2} l_{p}^{2} E^{2}\right)\right)\right]^{2} r_{+}^{2}(M-\varepsilon, Q-q) d \varepsilon}{\exp \left(\left(16 \pi \alpha^{2} l_{p}^{2} \varepsilon /(d-3)\left(2 r_{+}+\alpha l_{p}\right)\right)\left[1-\sqrt{1-4 \alpha^{2} l_{p}^{2}\left(1+l_{p}^{2} \beta^{2} r_{+}^{2}\right) /\left(2 r_{+}+\alpha l_{p}\right)^{2}}\right]^{-1}-1\right.} .
\end{aligned}
$$




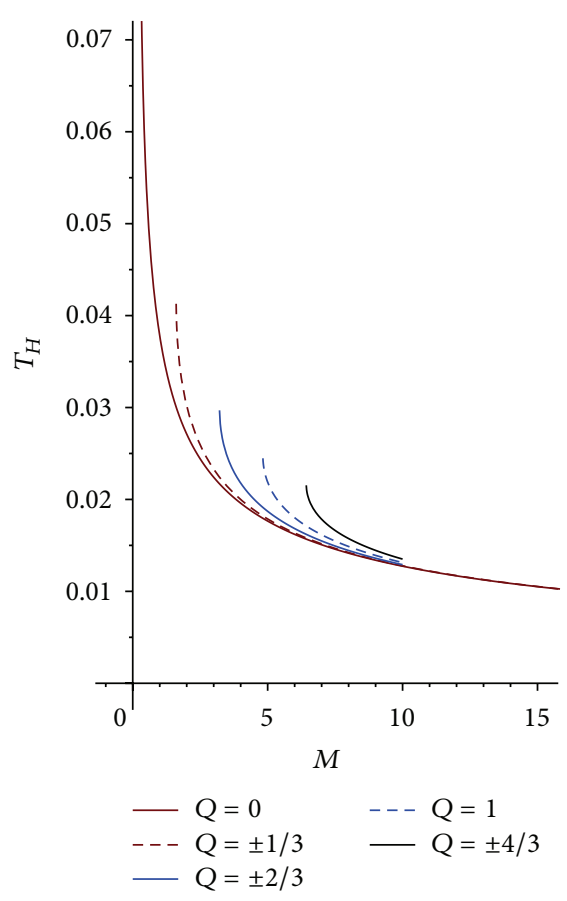

FIGURE 1: Hawking temperature for different amount of charges in presence of GUP.

It is important to remark that the total luminosities for microblack hole would be ten times bigger if we neglect backscattering effect. We are taking into account in the integration limits that the maximum energy of a radiated particle could be $M-M_{\mathrm{min}}^{\mathrm{GUP}}$. Equation (36) gives larger luminosities for smaller masses. The results show that, in large extra dimension scenario, Hawking temperature of charged black hole increases and leads to faster decay and less classical behaviors for black holes (Figure 2). On the other hand, it has been shown $[56,57]$ that the allowed particles forming the black hole at the LHC are quarks, antiquarks, and gluons which formed nine possible electric charge states: $\pm 4 / 3, \pm 1, \pm 2 / 3, \pm 1 / 3,0$. In this case, as far as the electric charge of the black hole increases, the minimum mass and its order of magnitude increase and the temperature peak is displaced to the lower temperature (see Figure 1). As (36) is related to the black hole temperature, based on the above arguments, the luminosity of charged nonrotating $\mathrm{TeV}$-scale black hole has different amount with respect to the charge of black hole and also extra dimensions.

\section{Conclusion and Discussion}

In this paper, we have investigated Hawking radiation of the charged massive particles as a semiclassical tunneling process from the charged nonrotating microblack hole. In this respect, we considered possible effect of natural cutoffs as a minimal length, a maximal momentum, and a minimal momentum on the tunneling rate. We have shown that, in the presence of generalized uncertainty principle, the tunneling rate of charged massive particle is deviated from thermal emission. In order to study the evolution of the TeV-scale

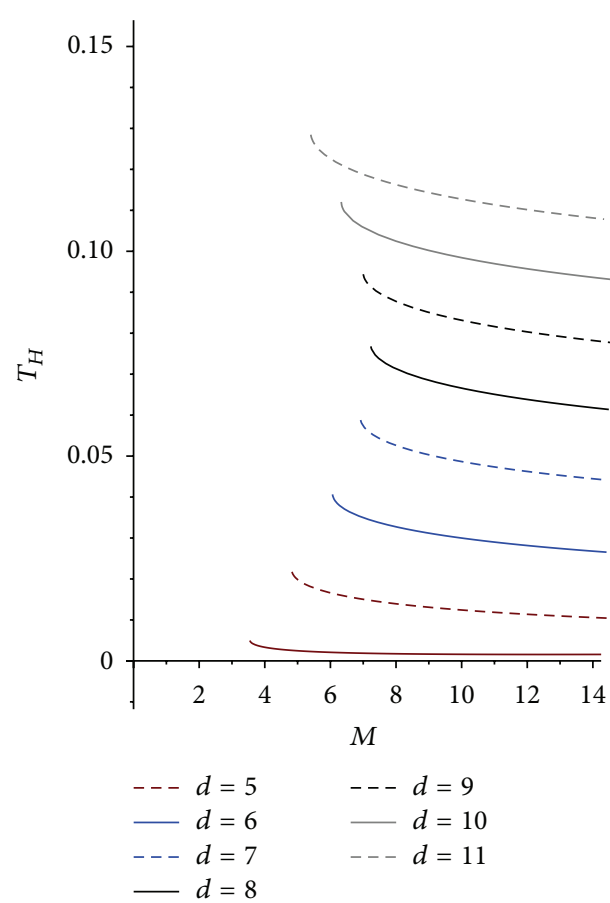

FIGURE 2: Hawking temperature with respect to the mass in terms of GUP.

microblack hole as it evaporates respecting energy and charge conservation, we have also modified the grey body factor, which allows considering the effect of the backscattered emitted radiation. We have calculated Hawking temperature based on the GUP which admitted a minimal length, a maximal momentum, and a minimal momentum. The adopted GUP predict a minimal mass remnant with respect to the charge of black hole. So we have been able to derive an expression for the luminosity that takes into account natural cutoffs in presence of large extra dimension based on ADD scenario for different amount of charge of black hole (Figures 2 and 1). The investigation implies that, considering natural cutoffs in the presence of LED, information conservation of charged nonrotating microblack hole is still possible.

\section{Competing Interests}

The authors declare that they have no competing interests.

\section{Acknowledgments}

M. J. Soleimani would like to give special thanks to E. C. Vagenas for his useful discussion. The paper is supported by University of Malaya (Grant no. Ru-023-2014) and University Kebangsaan Malaysia (Grant no. FRGS/2/2013/ST02/UKM/ $02 / 2$ coordinated by Dr. Geri Kibe Gopir).

\section{References}

[1] N. Arkani-Hame, S. Dimoulos, and G. Dvali, "The hierarchy problem and new dimensions at a millimeter," Physics Letters $B$, vol. 429, no. 3-4, pp. 263-272, 1998. 
[2] I. Antoniadis, N. Arkani-Hamed, S. Dimopoulos, and G. Dvali, "New dimensions at a millimeter to a fermi and superstrings at a TeV," Physics Letters B, vol. 436, no. 3-4, pp. 257-263, 1998.

[3] L. Randall and R. Sundrum, "Large mass hierarchy from a small extra dimension," Physical Review Letters, vol. 83, no. 17, pp. 3370-3373, 1999.

[4] L. Randall and R. Sundrum, "An alternative to compactification," Physical Review Letters, vol. 83, no. 23, pp. 4690-4693, 1999.

[5] P. C. Argyres, S. Dimopoulos, and J. March-Russell, "Black holes and sub-millimeter dimensions," Physics Letters B, vol. 441, no. 1-4, pp. 96-104, 1998.

[6] R. Emparan, G. T. Horowitz, and R. C. Myers, "Black holes radiate mainly on the brane," Physical Review Letters, vol. 85, no. 3, pp. 499-502, 2000.

[7] S. B. Giddings and S. Thomas, "Black hole bound on the number of species and quantum gravity at CERN LHC," Physical Review D, vol. 77, no. 4, Article ID 045027, 2008.

[8] J. L. Feng and A. D. Shapere, "Black hole production by cosmic rays," Physical Review Letters, vol. 88, no. 2, Article ID 021303, 4 pages, 2001.

[9] A. Ringwald and H. Tu, "Collider versus cosmic ray sensitivity to black hole production ", Physics Letters B, vol. 525, no. 1-2, pp. 135-142, 2002.

[10] M. Cavaglia, "Black hole and brane production in tev gravity: a review," International Journal of Modern Physics A, vol. 18, no. 11, p. 1843, 2003.

[11] G. Veneziano, "A stringy nature needs just two constants," Europhysics Letters (EPL), vol. 2, no. 3, pp. 199-204, 1986.

[12] A. Kempf, G. Mangano, and R. B. Mann, "Hilbert space representation of the minimal length uncertainty relation," Physical Review D, vol. 52, no. 2, pp. 1108-1118, 1995.

[13] A. Kempf and G. Mango, "Minimal length uncertainty relation and ultraviolet regularization," Physical Review D, vol. 55, no. 12, pp. 7909-7920, 1997.

[14] D. Amati, M. Ciafaloni, and G. Veneziano, "Can spacetime be probed below the string size?” Physics Letters B, vol. 216, no. 1-2, pp. 41-47, 1989.

[15] K. A. Meissner, "Black-hole entropy in loop quantum gravity," Classical and Quantum Gravity, vol. 21, no. 22, pp. 5245-5251, 2004.

[16] L. J. Gary, “Quantum gravity and minimum length," International Journal of Modern Physics A, vol. 10, no. 2, p. 145, 1995.

[17] F. Scardigli, "Generalized uncertainty principle in quantum gravity from micro-black hole gedanken experiment," Physics Letters B, vol. 452, no. 1-2, pp. 39-44, 1999.

[18] R. J. Adler, “Six easy roads to the Planck scale," American Journal of Physics, vol. 78, no. 9, pp. 925-932, 2010.

[19] A. F. Ali, S. Das, and E. C. Vagenas, "Proposal for testing quantum gravity in the lab," Physical Review D, vol. 48, no. 4, Article ID 044013, 2011.

[20] S. Das and E. C. Vagenas, "Universality of quantum gravity corrections," Physical Review Letters, vol. 101, no. 22, Article ID 221301, 2008.

[21] G. Amelino-Camelia, "Relativity in spacetimes with shortdistance structure governed by an observer-independent (Planckian) length scale," International Journal of Modern Physics D, vol. 11, no. 1, pp. 35-59, 2002.

[22] G. Amelino-Camelia, "Relativity: special treatment," Nature, vol. 418, no. 6893, pp. 34-35, 2002.
[23] G. Amelino-Camelia, "Doubly-special relativity: first results and key open problems," International Journal of Modern Physics D, vol. 11, no. 10, pp. 1643-1669, 2002.

[24] J. Kowalski-Glikman, "Introduction to doubly special relativity," in Planck Scale Effects in Astrophysics and Cosmology, vol. 669, pp. 131-159, Springer, Berlin, Germany, 2005.

[25] M. K. Parikh and F. Wilczek, "Hawking radiation as tunneling," Physical Review Letters, vol. 85, no. 24, pp. 5042-5045, 2000.

[26] M. Maggiore, "A generalized uncertainty principle in quantum gravity," Physics Letters B, vol. 304, no. 1-2, pp. 65-69, 1993.

[27] M. Maggiore, "The algebraic structure of the generalized uncertainty principle," Physics Letters B, vol. 319, no. 1-3, pp. 83-86, 1993.

[28] J. Magueijo and L. Smolin, "Generalized Lorentz invariance with an invariant energy scale," Physical Review D, vol. 67, no. 4, Article ID 044017, 12 pages, 2003.

[29] J. L. Corte's and J. Gamba, "Quantum uncertainty in doubly special relativity," Physical Review D, vol. 71, no. 6, Article ID 065015, 2005.

[30] J. Magueijo and L. Smolin, "Lorentz invariance with an invariant energy scale," Physical Review Letters, vol. 88, no. 19, Article ID 190403, 4 pages, 2002.

[31] J. Magueijo and L. Smolin, "String theories with deformed energy-momentum relations, and a possible nontachyonic bosonic string," Physical Review D, vol. 71, no. 2, Article ID 026010, 6 pages, 2005.

[32] H. Hinrichsen and A. Kempf, "Maximal localization in the presence of minimal uncertainties in positions and in momenta," Journal of Mathematical Physics, vol. 37, no. 5, pp. 2121-2137, 1996.

[33] M. Zarei and B. Mirza, "Minimal uncertainty in momentum: the effects of IR gravity on quantum mechanics," Physical Review D, vol. 79, Article ID 125007, 2009.

[34] P. Pedram, K. Nozari, and S. H. Taheri, "The effects of minimal length and maximal momentum on the transition rate of ultra cold neutrons in gravitational field," Journal of High Energy Physics, vol. 2011, article 93, 2011.

[35] R. C. Myers and M. J. Perry, "Black holes in higher dimensional space-times," Annals of Physics, vol. 172, no. 2, pp. 304-347, 1986.

[36] Q.-Q. Jiang and S.-Q. Wu, "Hawking radiation of charged particles as tunneling from Reissner-Nordström-de Sitter black holes with a global monopole," Physics Letters B, vol. 635, no. 2-3, pp. 151-155, 2006.

[37] J. Y. Zhang and Z. Zhao, "Hawking radiation of charged particles via tunneling from the Reissner-Nordström black hole," Journal of High Energy Physics, vol. 2005, no. 10, article 055, 2005.

[38] J. Mäkelä and P. Repo, "Quantum-mechanical model of the Reissner-Nordström black hole," Physical Review D, vol. 57, no. 8, pp. 4899-4916, 1998.

[39] K. Srinivasan and T. Padmanban, "Particle production and complex path analysis," Physical Review D, vol. 60, no. 2, Article ID $024007,1999$.

[40] J. L. Feng, A. Rajaraman, and F. Takayama, "Probing gravitational interactions of elementary particles," International Journal of Modern Physics D, vol. 13, no. 10, pp. 2355-2359, 2004.

[41] K. Nozari and S. Saghafi, "Parikh-Wilczek tunneling from noncommutative higher dimensional black holes," Journal of High Energy Physics, vol. 2012, no. 11, article 005, 2012.

[42] P. Kraus and F. Wilczek, "Self-interaction correction to black hole radiance," Nuclear Physics B, vol. 433, no. 2, pp. 403-420, 1995. 
[43] E. C. Vagenas, "Are extremal 2D black holes really frozen?" Physics Letters B, vol. 503, no. 3-4, pp. 399-403, 2001.

[44] E. C. Vagenas, "Two-dimensional dilatonic black holes and Hawking radiation," Modern Physics Letters A, vol. 17, no. 10, pp. 609-618, 2002.

[45] E. C. Vagenas, "Semiclassical corrections to the BekensteinHawking entropy of the BTZ black hole via self-gravitation," Physics Letters B, vol. 533, no. 3-4, pp. 302-306, 2002.

[46] A. J. M. Medved, "Radiation via tunnelling in the charged BTZ black hole," Classical and Quantum Gravity, vol. 19, no. 3, article 589, 2002

[47] E. C. Vagenas, "Generalization of the KKW analysis for black hole radiation," Physics Letters B, vol. 559, no. 1-2, pp. 65-73, 2003.

[48] M. Parikh, "A secret tunnel through the horizon," International Journal of Modern Physics D, vol. 13, no. 10, pp. 2351-2354, 2004.

[49] E. T. Akhmedov, V. Akhmedova, and D. Singleton, "Hawking temperature in the tunneling picture," Physics Letters B, vol. 642, no. 1-2, pp. 124-128, 2006.

[50] S.-Q. Wu and Q.-Q. Jiang, "Hawking radiation of charged particles as tunneling from higher dimensional reissner-nordstromde sitter black holes," https://arxiv.org/abs/hep-th/0603082.

[51] S. W. Hawking, "Particle creation by black holes," Communications in Mathematical Physics, vol. 43, no. 3, pp. 199-220, 1975.

[52] R. J. Adler, P. Chen, and D. I. Santiago, "The generalized uncertainty principle and black hole remnants," General Relativity and Gravitation, vol. 33, no. 12, pp. 2101-2108, 2001.

[53] S. K. Modak, "Backreaction due to quantum tunneling and modification to the black hole evaporation process," Physical Review D, vol. 90, no. 4, Article ID 044015, 2014.

[54] R. Torres, F. Fayos, and O. Lorente-Espín, "Evaporation of (quantum) black holes and energy conservation," Physics Letters B, vol. 720, no. 1-3, pp. 198-204, 2013.

[55] A. Fabbri and J. Navario-Salas, Modeling Black Hole Evaporation, Imperial College Press, London, UK, 2005.

[56] D. M. Gingrich, "Quantum black holes with charge, color and spin at the LHC," Journal of Physics G: Nuclear and Particle Physics, vol. 37, no. 10, Article ID 105008, 2010.

[57] G. Landsberg, "Black holes at future colliders and beyond," Journal of Physics G, vol. 32, no. 9, p. 337, 2006. 

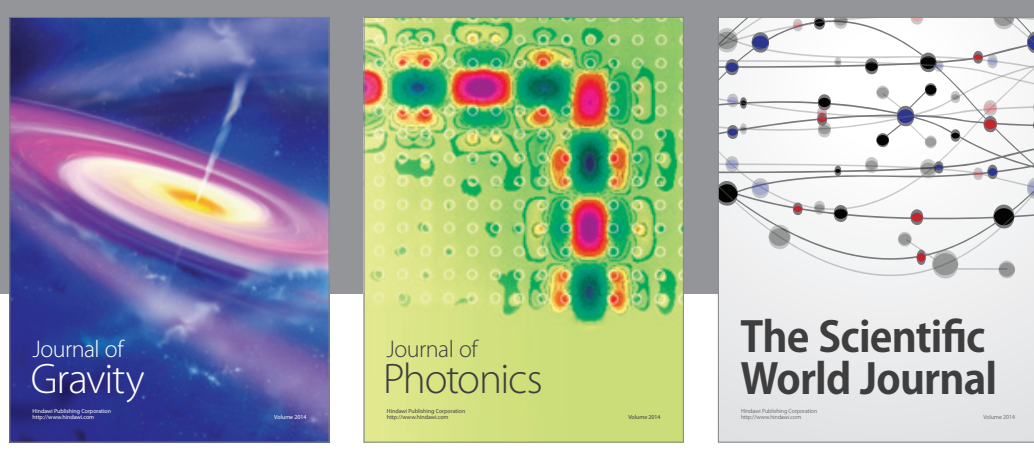

The Scientific World Journal
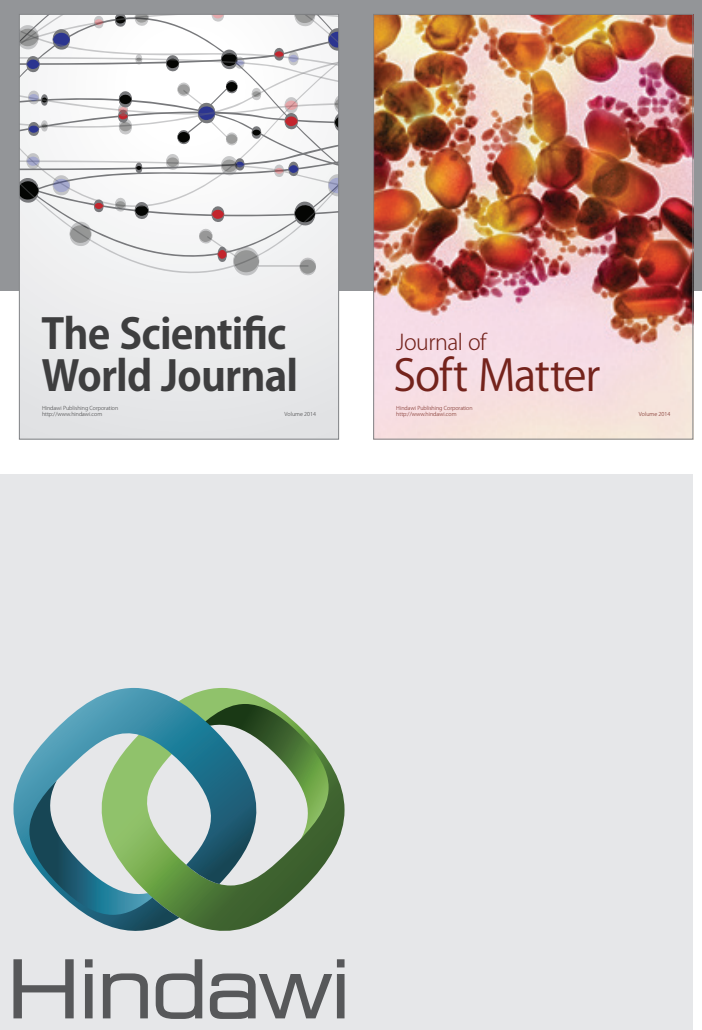

Submit your manuscripts at

http://www.hindawi.com

nternational Journal of

Statistical Mechanics
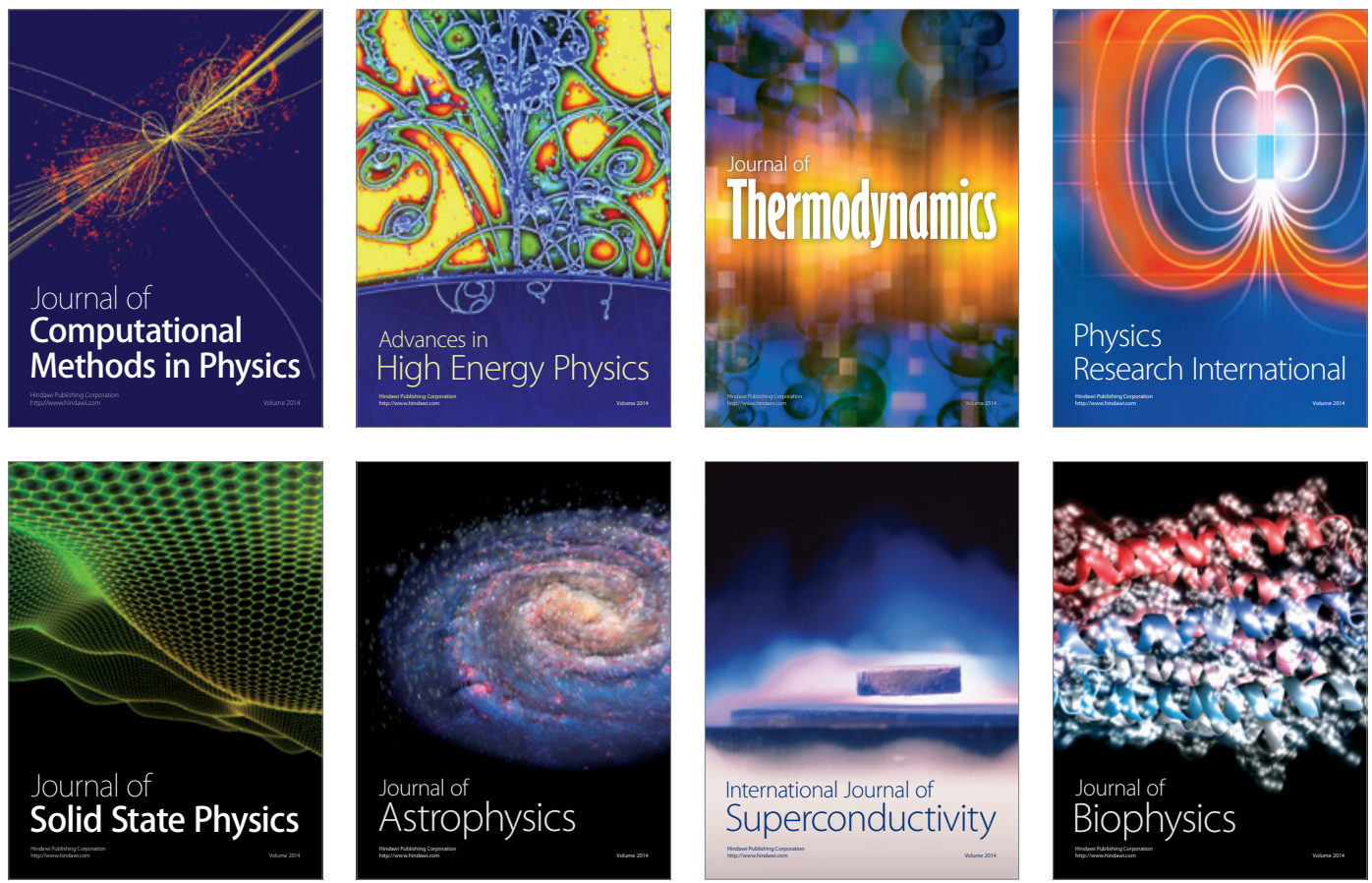
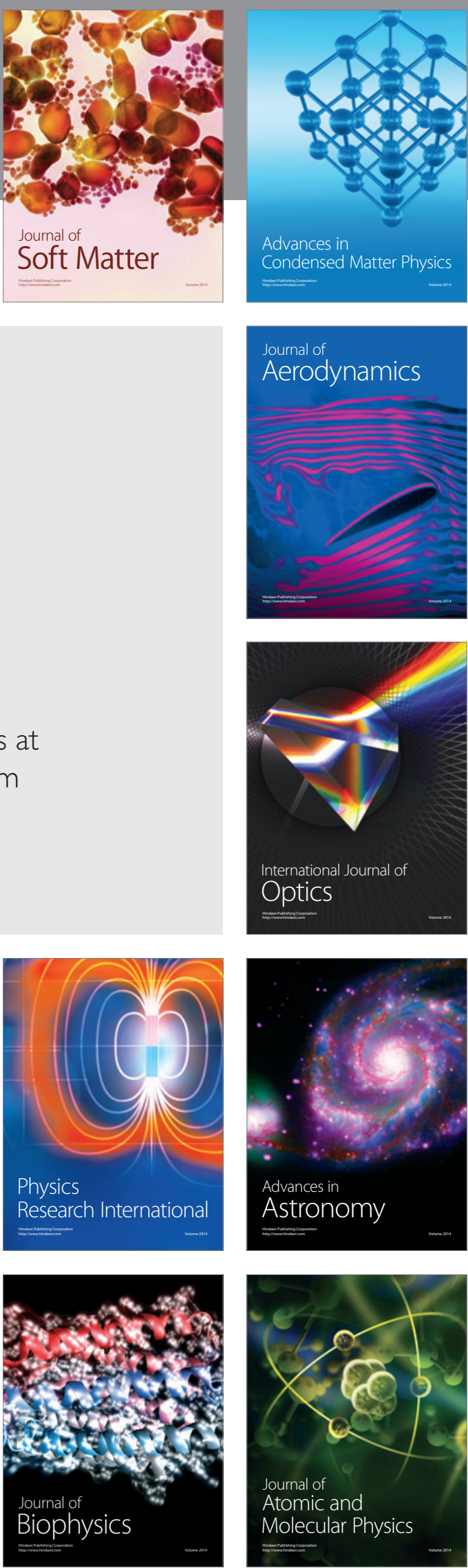